June 22, 2001

KEK-TH-772

TOHO-FP-0169

\title{
Classical AdS Superstring Mechanics
}

\author{
Machiko Hatsudał and Kiyoshi Kamimura $\left.\right|^{\dagger}$ \\ Theory Division, High Energy Accelerator Research Organization (KEK), \\ Tsukuba, Ibaraki, 305-0801, Japan \\ $\dagger$ Department of Physics, Toho University, Funabashi, 274-8510, Japan
}

\begin{abstract}
We analyze the anti-de Sitter (AdS) superparticle and superstring systems described in terms of supermatrix valued coordinates proposed by Roiban and Siegel. This approach gives simple symmetry transformations and equations of motion. We examine their $\kappa$-transformations, infinite reducibility and $\kappa$-gauge fixing conditions. A closed first class constraint set for the AdS superparticle is $G L(4 \mid 4)$ covariant and keeping superconformal symmetry manifestly. For the AdS superstring $\sigma$-dependence breaks the $G L(4 \mid 4)$ covariance, where supercovariant derivatives and currents satisfy the inhomogeneous $G L(4 \mid 4)$. A closed first class constraint set for the AdS superstring turns out to be the same as the one for a superstring in flat space, namely $\mathcal{A B C D}$ constraints.
\end{abstract}

PACS: 11.30.Pb;11.17.+y;11.25.-w

Keywords: Superalgebra; Anti-de Sitter; coset construction;

\footnotetext{
${ }^{1}$ mhatsuda@post.kek.jp

${ }^{2}$ kamimura@ph.sci.toho-u.ac.jp
} 


\section{Introduction}

The AdS/CFT correspondence conjectured by [1] requires appropriate descriptions of the superstring theories in anti-de Sitter (AdS) spaces corresponding to the conformal field theories (CFT). Since AdS spaces contain the Ramond-Ramond flux, the manifest space-time supersymmetric Green-Schwarz formalism is more suitable than the NeveuSchwarz-Ramond formalism. The superstring theory in the $\operatorname{AdS}_{5} \times \mathrm{S}^{5}$ background, known to be dual of the four dimensional CFT, is investigated at the classical level [2, 3, 5] and at the semi-classical level [4] in the Green-Schwarz formalism. The global symmetry of the $\mathrm{AdS}_{5} \times \mathrm{S}^{5}$ space is $S U(2,2 \mid 4)$. The AdS superstring action is constructed based on a coset $S U(2,2 \mid 4) /(S O(4,1) \times S O(5))$ [2]. Recently an alternative approach has been proposed by Roiban and Siegel [6] based on a coset $G L(4 \mid 4) /(S p(4) \times G L(1))^{2}$, which is obtained by Wick rotations and introducing scaling degrees of freedom into $S U(2,2 \mid 4) /(S O(4,1) \times S O(5))$. In this alternative approach the coordinate is supermatrix valued. The Lobachevski metric follows from this matrix parametrization rather than the conventional exponential parametrization. In this paper we follow this approach to examine the classical mechanics of the AdS superparticle and superstring.

When an element of the coset is given as $Z_{M}^{A} \in G L(4 \mid 4) /(S p(4) \times G L(1))^{2}$, the right invariant and left invariant one-forms are constructed as $\left(d Z Z^{-1}\right)_{M}^{N}$ and $\left(Z^{-1} d Z\right)_{A}^{B}$ respectively. If we introduce its canonical conjugates, $P_{A}{ }^{M}$, the global symmetry generators and the supercovariant derivatives can be written as $G_{M}^{N}=(Z P)_{M}^{N}$ and $D_{A}^{B}=(P Z)_{A}{ }^{B}$ respectively as we will see in next section. The global symmetry generators $G L(4 \mid 4) \ni G_{M N}$ satisfy

$$
\left[G_{M N}, G_{L K}\right]=-\Omega_{L N} G_{M K}+\Omega_{M K} G_{L N}
$$

with an antisymmetric metric $\Omega$ which raises and lowers the indices. On the other hand the supercovariant derivatives satisfy

$$
\left[D_{A B}, D_{C D}\right]=\Omega_{C B} D_{A D}-\Omega_{A D} D_{C B} .
$$

The $D$ 's correspond to $\tau$ development, so the supercovariant derivative governs the AdS superparticle. The covariant derivatives commute/anticommute with the global charges, $\left[G_{M N}, D_{A B}\right]=0$. The fermionic components of supercovariant derivatives are nothing but the fermionic constraints whose first class part generate $\kappa$-symmetries. For a flat superparticle supercovariant derivatives are $p_{\mu}$ and $d_{\alpha}$, and the closed first class constraint set is given by bi-linear forms of covariant derivatives, $p^{\mu} p_{\mu}=0$ and $p_{\mu} \gamma^{\mu} d=0$ [7, 8, 9]. For a conformal particle covariant derivatives are conformal group elements, and the closed

first class constraint set is obtained by squaring the covariant derivatives [10, 11]. The AdS superparticle is also the case where a sufficient first class constraint set is expressed as

$$
\left(D^{2}\right)_{A}{ }^{C}=D_{A}{ }^{B} D_{B}{ }^{C}=0 .
$$

They satisfy the following algebra

$$
\left[D^{2}{ }_{A B}, D_{C D}^{2}\right]=D_{A D}^{3} \Omega_{C B}-D_{C B}^{3} \Omega_{A D}+D_{A D}^{2} D_{C B}-D_{C B}^{2} D_{A D}
$$


and are reducible,

$$
D_{A}{ }^{B} D^{2}{ }_{B}^{C}-D_{A}^{2}{ }^{B} D_{B}{ }^{C}=0 \text {. }
$$

The first class constraints (1.3) includes the massless equation and $\kappa$-generators representing local symmetries for the AdS superparticle. Gauge fixing follows to the form of the first class constraints.

For an AdS superstring the $\sigma$ dependence requires $\sigma$ direction current $J_{A B}$ and the system is described in terms of $D$ and $J$ satisfying

$$
\begin{aligned}
{\left[D_{A B}, D_{C D}\right] } & =\Omega_{C B} D_{A D}-\Omega_{A D} D_{C B} \\
{\left[D_{A B}, J_{C D}\right] } & =\Omega_{C B} J_{A D}-\Omega_{A D} J_{C B} \\
{\left[J_{A B}, J_{C D}\right] } & =0 .
\end{aligned}
$$

A superstring in flat space is described by left/right $\left(\partial_{\tau} \pm \partial_{\sigma}\right)$ chiral representations, but this is not the case. The naive left/right combination $D \pm J$ does not show separation into two sets, but

$$
\begin{aligned}
& {\left[(D \pm J)_{A B},(D \pm J)_{C D}\right]=\Omega_{C B}(D \pm 2 J)_{A D}-\Omega_{A D}(D \pm 2 J)_{C B}} \\
& {\left[(D \pm J)_{A B},(D \mp J)_{C D}\right]=\Omega_{C B} D_{A D}-\Omega_{A D} D_{C B} .}
\end{aligned}
$$

The naive replacement $D \rightarrow D \pm J$ in (1.3)-(1.5) does not work as the stringy extension of the local symmetry constraint set. To find the local symmetry constraint set is not trivial as in the AdS superparticle case, and one needs to start with the $\kappa$-invariant action for the AdS superstring.

It is interesting that the algebra (1.6) is recognized as an inhomogeneous $G L(4 \mid 4)$ and it is obtained from the following algebra

$$
\begin{aligned}
{\left[D_{A B}, D_{C D}\right] } & =\Omega_{C B} D_{A D}-\Omega_{A D} D_{C B} \\
{\left[D_{A B}, J_{C D}\right] } & =\Omega_{C B} J_{A D}-\Omega_{A D} J_{C B} \\
{\left[J_{A B}, J_{C D}\right] } & =\Omega_{C B} D_{A D}-\Omega_{A D} D_{C B}
\end{aligned}
$$

by the Inönü-Wigner contraction [12] with $J \rightarrow R J$ and $R \rightarrow \infty$ limit. In contrast to (1.7) the left/right chiral separation is possible for $R=1$,

$$
\begin{aligned}
& {\left[(D \pm J)_{A B},(D \pm J)_{C D}\right]=\Omega_{C B}\left\{\left(1+\frac{1}{R^{2}}\right) D \pm 2 J\right\}_{A D}-\Omega_{A D}\left\{\left(1+\frac{1}{R^{2}}\right) D \pm 2 J\right\}_{C B}} \\
& {\left[(D \pm J)_{A B},(D \mp J)_{C D}\right]=\Omega_{C B}\left(1-\frac{1}{R^{2}}\right) D_{A D}-\Omega_{A D}\left(1-\frac{1}{R^{2}}\right) D_{C B}}
\end{aligned}
$$

The AdS superstring case (1.7) is given for a case with $R \rightarrow \infty$. In this case rescaling ambiguity of $J$ is remained. We will construct the left/right chiral algebra up to constraints in the section 4 where chiral combinations are $D_{\text {bose }} \pm J_{\text {bose }}$ and $D_{\text {fermi }} \pm(1 / 2) J_{\text {fermi }}$.

A local symmetry constraint set for the AdS superstring is constructed and compared with the one for a superstring in a flat space, namely $\mathcal{A B C D}$ constraints [7, 8, 9]. A 
flat superstring system can be described in two approaches. One is the usual canonical approach that includes both first class and second class constraints, and products of two constraints can be set to be zero consistently [13. Another one is the first class approach that includes only first class constraints and square of the supercovariant derivatives are taken as first class constraints [7]. It is shown that these approaches, [13] and [7], give the same physical states by analyzing in the light-cone gauge [14]. An advantage of this first class approach is to avoid complicated second class constraints. Disadvantage is that the first class constraints are infinitely reducible, so practical covariant quantum computation is difficult [15. In this paper we will focus on the classical mechanics for AdS objects to clarify common features and different features from the ones in the flat background.

The organization of this paper is as follows. In section 2, we perform the canonical analysis of the AdS superparticle system. The $\kappa$ symmetry constraints and its reducibility are clarified. The equation of motion is also given, and it has simple free form. In section 3 , we confirm the necessary conditions of the closed first class constraint set by direct computation from an action. The $\left(D^{2}\right)_{A}^{B}=0$ constraint set is analyzed in "light-conelike" gauge that suppresses half of fermionic degrees of freedom, and then we show that $\left(D^{2}\right)_{A}^{B}=0$ constraints reduce into $\mathrm{GL}(2) \times \mathrm{GL}(4) \cong \mathrm{U}(2) \times \mathrm{U}(4)$ generators which turn out to represent the second class constraints. In other words, the AdS superparticle with first class constraint set, $\left(D^{2}\right)_{A}^{B}=0$, is equal to the system with the first and second class constraints. In section 4, the AdS superstring system is examined in analogous to the AdS superparticle case. The $\kappa$-gauge fixing and equations of motions are examined, and we show that the complex gauge proposed in [6] can not be applied. The closed first class constraint set is shown to correspond to the one in a flat background, namely $\mathcal{A B C D}$ constraints.

\section{Classical AdS superparticle mechanics}

A supersymmetric object propagating in $\mathrm{AdS}_{5} \times \mathrm{S}^{5}$ space is described using by the following coset superspace and the coset elements are parameterized including quotient parameters as 6]

$$
\frac{G L(4 \mid 4)}{(S p(4) \times G L(1))^{2}} \ni Z_{M}{ }^{A} \quad, \quad Z_{M}{ }^{A}=\left(\begin{array}{cc}
Z_{m}{ }^{a} & Z_{m}{ }^{\bar{a}} \\
Z_{\bar{m}}{ }^{a} & Z_{\bar{m}}{ }^{\bar{a}}
\end{array}\right)=\left(\begin{array}{cc}
X_{m}{ }^{a} & \Theta_{m}{ }^{\bar{a}} \\
\bar{\Theta}_{\bar{m}}{ }^{a} & \bar{X}_{\bar{m}}{ }^{\bar{a}}
\end{array}\right),
$$

where $M$ and $A$ are transformed by global $G L(4 \mid 4)$ and local $(S p(4) \times G L(1))^{2}$ corresponding to the global coordinate transformations and the local Lorentz and the dilatation respectively. Throughout this paper we put full components of parameters $8^{2}=64$ as $Z_{M}{ }^{A}$, then gauge degrees of freedom are eliminated by constraints. The left invariant current is

$$
J_{A}^{B}=Z_{A}^{-1}{ }^{M} d Z_{M}^{B} .
$$

A $G L(4)$ matrix $M_{a b}$ is decomposed into a trace part, symmetric part and tracelessantisymmetric part

$$
M_{a b}=-\frac{1}{4} \Omega_{a b} M_{c}^{c}+M_{(a b)}+M_{\langle a b\rangle}=-\frac{1}{4} \Omega \operatorname{tr} M+(M)+\langle M\rangle,
$$


where $\Omega$ is antisymmetric $S P(4)$ metric raising and lowering indices in the NW rule. The coset parts of the left invariant currents are $J_{\langle a b\rangle}, J_{\langle\bar{a} \bar{b}\rangle}, J_{a \bar{b}}$ and $J_{a \bar{b}}$, and local Lorentz $S p(4)$, dilatation $G L(1)$ connections are $J_{(a b)}, \operatorname{tr} J$ respectively.

The action for a superparticle is given in terms of the 1 -form currents $J_{A B}=d \sigma^{\mu} J_{\mu A B}$;

$$
S=-\int d \tau \frac{1}{2 e}\left(J_{0}^{\langle a b\rangle} J_{0}\langle a b\rangle-J_{0}^{\langle\bar{a} \bar{b}\rangle} J_{0}\langle\bar{a} \bar{b}\rangle\right)=\int d \tau \mathcal{L}
$$

Canonical momentum for $Z_{M}{ }^{A}$ is defined as

$$
P_{A}{ }^{M}=\frac{\delta^{r} S}{\delta \dot{Z}_{M}{ }^{A}}(-)^{A}=\left(\begin{array}{ccc}
P_{a}{ }^{m} & \bar{\zeta}_{a} & \bar{m} \\
\zeta_{\bar{a}} & \bar{P}_{\bar{a}} & \bar{m}
\end{array}\right),
$$

and the Hamiltonian is given by

$$
\mathcal{H}=\left[\sum P_{A}{ }^{M} \dot{Z}_{M}{ }^{A}(-)^{A}-\mathcal{L}\right]
$$

Corresponding to (2.5) we choose the canonical poisson bracket as

$$
\left[Z_{M}^{A}, P_{B}^{N}\right]=(-1)^{A} \delta_{B}^{A} \delta_{M}^{N}
$$

in order to preserve supertrace

$$
\begin{aligned}
& \mathrm{S} \operatorname{Tr} f g=\sum f^{A M} g_{M A}(-)^{A}, \\
& {[\mathrm{~S} \operatorname{Tr}(f Z), \mathrm{S} \operatorname{Tr}(P g)]=-\mathrm{S} \operatorname{Tr}(f g) .}
\end{aligned}
$$

The AdS superparticle action (2.4) is rewritten as

$$
\begin{aligned}
S= & -\int d \tau \frac{1}{2 e} \operatorname{tr}\left(\left\langle X^{-1} v\left(\dot{X}-\Theta \bar{X}^{-1} \dot{\bar{\Theta}}\right)\right\rangle\left\langle X^{-1} v\left(\dot{X}-\Theta \bar{X}^{-1} \dot{\bar{\Theta}}\right)\right\rangle\right. \\
& \left.-\left\langle\bar{X}^{-1} \bar{v}\left(\dot{\bar{X}}-\bar{\Theta} X^{-1} \dot{\Theta}\right)\right\rangle\left\langle\bar{X}^{-1} \bar{v}\left(\dot{\bar{X}}-\bar{\Theta} X^{-1} \dot{\Theta}\right)\right\rangle\right) \\
v & =\left(I-\Theta \bar{X}^{-1} \bar{\Theta} X^{-1}\right)^{-1} \\
\bar{v} & =\left(I-\bar{\Theta} X^{-1} \Theta \bar{X}^{-1}\right)^{-1}
\end{aligned}
$$

Canonical conjugates are

$$
\begin{aligned}
P_{a}^{m} & \equiv \frac{\delta S}{\delta \dot{X}_{m}^{a}}=-\frac{1}{e} X^{-1} v\left(\dot{X}-\Theta \bar{X}^{-1} \dot{\bar{\Theta}}\right) X^{-1} v \\
\bar{P}_{\bar{a}}{ }^{\bar{m}} & \equiv-\frac{\delta S}{\delta \dot{\bar{X}}_{\bar{m}}{ }^{\bar{a}}}=-\frac{1}{e} \bar{X}^{-1} \bar{v}\left(\dot{\bar{X}}-\bar{\Theta} X^{-1} \dot{\Theta}\right) \bar{X}^{-1} \bar{v} \\
\zeta_{\bar{a}}{ }^{m} & \equiv-\frac{\delta^{r} S}{\delta \dot{\Theta}_{m}{ }^{a}}=-\bar{P} \bar{\Theta} X^{-1} \\
\bar{\zeta}_{a}{ }^{\bar{m}} & \equiv \frac{\delta^{r} S}{\delta \dot{\bar{\Theta}}_{\bar{m}}^{a}}=-P \Theta \bar{X}^{-1} .
\end{aligned}
$$


The supercovariant derivatives are

$$
\begin{aligned}
& D_{A}{ }^{B}=P_{A}{ }^{M} Z_{M}{ }^{B}=\left(\begin{array}{ll}
\mathbf{D}_{a}{ }^{b} & D_{a} \bar{b} \\
\bar{D}_{\bar{a}}{ }^{b} & \overline{\mathbf{D}}_{\bar{a}} \bar{b}
\end{array}\right), \\
& \mathbf{D} \equiv P X+\bar{\zeta} \bar{\Theta}, \overline{\mathbf{D}} \equiv \bar{P} \bar{X}+\zeta \Theta \\
& D \equiv P \Theta+\bar{\zeta} \bar{X}, \bar{D} \equiv \bar{P} \bar{\Theta}+\zeta X
\end{aligned}
$$

generating right $G L(4 \mid 4)$ transformations

$$
\delta_{\Lambda} Z_{M}{ }^{A}=\left[Z_{M}{ }^{A}, \mathrm{~S} \operatorname{Tr} D \Lambda\right]=(Z \Lambda)_{M}{ }^{A} .
$$

Its subgroups $G L(1), S p(4), \overline{G L(1)}, \overline{S p(4)}$ are local gauge symmetries, and their generators are set to be constraints in our approach

$$
\operatorname{tr} \mathbf{D}=(\mathbf{D})=\operatorname{tr} \overline{\mathbf{D}}=(\overline{\mathbf{D}})=0 .
$$

The last two fermionic equations of (2.10) are primary constraints,

$$
D_{a}{ }^{\bar{b}}=0, \quad \bar{D}_{\bar{a}}{ }^{b}=0
$$

satisfying following poisson brackets

$$
\left[D_{a}{ }^{\bar{b}}, \bar{D}_{\bar{c}}{ }^{d}\right]=\delta_{a}^{d} \overline{\mathbf{D}}_{\bar{c}}{ }^{\bar{b}}-\delta_{\bar{c}}^{\bar{b}} \mathbf{D}_{a}{ }^{d},[D, D]=[\bar{D}, \bar{D}]=0 .
$$

The Hamiltonian (2.6) is obtained as

$$
\begin{aligned}
\mathcal{H} & =-e A_{\mathrm{P}}+\operatorname{tr}[D \bar{\lambda}+\bar{D} \lambda] \\
A_{\mathrm{P}} & =\frac{1}{2} \operatorname{tr}\left[\langle\mathbf{D}\rangle^{2}-\langle\overline{\mathbf{D}}\rangle^{2}\right]=0
\end{aligned}
$$

where $A_{\mathrm{P}}=0$ is a secondary constraint for $\dot{\Pi}_{e}=0$ and multipliers determined from consistency as $\lambda=\bar{\lambda}=0$. Because of the bosonic constraint (2.17) the rank of the right hand side of (2.15) is half the number of its maximal rank, and whose zero modes are given as

$$
\left(\delta_{a}^{d} \overline{\mathbf{D}}_{\bar{c}}^{\bar{b}}-\delta_{\bar{c}}^{\bar{b}} \mathbf{D}_{a}{ }^{d}\right)\left(\delta_{d}^{e} \overline{\mathbf{D}}_{\bar{b}}{ }^{\bar{f}}+\delta_{\bar{b}}^{\bar{f}} \mathbf{D}_{d}{ }^{e}\right)=\frac{1}{2} \delta_{a}^{e} \delta_{\bar{c}}^{\bar{f}} A_{\mathrm{P}} \approx 0,
$$

where equality holds up to gauge constraints (2.13). Half of the fermionic constraints are first class and another half are second class constraints as in a flat superparticle. The fermionic constraints $D$ and $\bar{D}$ in (2.14) are projected into first class constraints using $\langle\mathbf{D}\rangle$ and $\langle\overline{\mathbf{D}}\rangle$ as

$$
\begin{aligned}
\mathcal{B}_{\mathrm{P} a}{ }^{\bar{b}} & =\langle\mathbf{D}\rangle_{a}{ }^{b} D_{b}{ }^{\bar{b}}+D_{a}{ }^{\bar{a}}\langle\overline{\mathbf{D}}\rangle_{\bar{a}}{ }^{\bar{b}} \\
\overline{\mathcal{B}}_{\mathrm{P}}{ }^{b}{ }^{b} & =\langle\overline{\mathbf{D}}\rangle_{\bar{a}}{ }^{\bar{b}} \bar{D}_{\bar{b}}{ }^{b}+\bar{D}_{\bar{a}}{ }^{a}\langle\mathbf{D}\rangle_{a}{ }^{b} .
\end{aligned}
$$

They generate following $\kappa$-transformations

$$
\begin{aligned}
\delta_{\kappa, \bar{\kappa}} Z & =[Z, \operatorname{tr}(\overline{\mathcal{B}} \kappa-\mathcal{B} \bar{\kappa})] \\
\delta_{\kappa, \bar{\kappa}} X & =\Theta(\bar{\kappa}\langle\mathbf{D}\rangle+\langle\overline{\mathbf{D}}\rangle \bar{\kappa}) \\
\delta_{\kappa, \bar{\kappa}} \bar{X} & =\bar{\Theta}(\kappa\langle\overline{\mathbf{D}}\rangle+\langle\mathbf{D}\rangle \kappa) \\
\delta_{\kappa, \bar{\kappa}} \Theta & =X(\kappa\langle\overline{\mathbf{D}}\rangle+\langle\mathbf{D}\rangle \kappa) \\
\delta_{\kappa, \bar{\kappa}} \bar{\Theta} & =\bar{X}(\bar{\kappa}\langle\mathbf{D}\rangle+\langle\overline{\mathbf{D}}\rangle \bar{\kappa}) .
\end{aligned}
$$


Both parameters $\kappa$ and $\bar{\kappa}$ have following zero modes, and this reducibility continues infinitely

$$
\begin{aligned}
& \delta \kappa=\langle\mathbf{D}\rangle \kappa_{1}-\kappa_{1}\langle\overline{\mathbf{D}}\rangle, \quad \delta \kappa_{1}=\langle\mathbf{D}\rangle \kappa_{2}+\kappa_{2}\langle\overline{\mathbf{D}}\rangle, \quad \cdots \\
& \delta \bar{\kappa}=\langle\overline{\mathbf{D}}\rangle \bar{\kappa}_{1}-\bar{\kappa}_{1}\langle\mathbf{D}\rangle, \quad \delta \bar{\kappa}_{1}=\langle\overline{\mathbf{D}}\rangle \bar{\kappa}_{2}+\bar{\kappa}_{2}\langle\mathbf{D}\rangle, \quad \cdots
\end{aligned}
$$

where $A_{\mathrm{P}}=0$ is used. This fact leads to that only each half components of $\Theta$ and $\bar{\Theta}$ can be fixed by using $\kappa$-symmetries. Therefore the complex gauge proposed in the literature [6] can not be imposed for the AdS superparticle.

Classical equations of motion are given by this Hamiltonian with for example $e=1$

$$
\dot{Z}_{M}{ }^{A}=\left[Z_{M}{ }^{A}, \mathcal{H}\right]=Z_{M}^{B} \Gamma_{\mathrm{P} B}{ }^{A}, \quad \Gamma_{\mathrm{P}}=\left(\begin{array}{cc}
\langle\mathbf{D}\rangle & 0 \\
0 & \langle\overline{\mathbf{D}}\rangle
\end{array}\right)
$$

where the fermionic constraints (2.14) is used. The second derivative of $Z$ leads to

$$
\ddot{Z}-\dot{Z} \Gamma_{\mathrm{P}}=0
$$

and it suggests that $\Gamma_{\mathrm{P}}$ is interpreted as the induced connection onto the world line. $\langle\mathbf{D}\rangle$ and $\langle\overline{\mathbf{D}}\rangle$, are conserved "momenta" of this system. They satisfy

$$
\left[\langle\mathbf{D}\rangle_{\langle a b\rangle},\langle\mathbf{D}\rangle_{\langle c d\rangle}\right]=\Omega_{\langle c|\langle b}(\mathbf{D})_{a\rangle|d\rangle}-\Omega_{\langle a|\langle d}(\mathbf{D})_{c\rangle|b\rangle}
$$

which is the AdS momentum algebra, and the similar form for bared $\left(\mathrm{S}^{5}\right)$ sector. (2.22) represents a free particle.

Solutions of (2.22) are

$$
Z(\tau)=Z_{0} e^{\Gamma_{\mathrm{P}} \tau}
$$

The world line element is calculated as

$$
d s^{2}=\left.\operatorname{STr} J_{0}^{2}\right|_{\text {bosonic part }}=\operatorname{STr}\left(Z^{-1} \dot{Z}\right)^{2}=\operatorname{STr} \Gamma_{\mathrm{P}}{ }^{2}=\frac{1}{2} A_{\mathrm{P}}=0
$$

where the constraints (2.17) is used. Therefore the free superparticle moves along the null geodesic of the $\mathrm{AdS}(\mathrm{S})$ space.

\section{Local symmetries of the AdS superparticle}

Local symmetries are expressed by a closed first class constraint set. In the previous section we perform a canonical analysis of a superparticle where products of constraints including second class are set to be zero consistently. In this section we examine an alternative approach that contains only first class constraints without second class constraints. We obtain a closed first class constraint set and then examine the physical states in a "light-cone-like" gauge comparing with the one described by the previous approach. 
Although we know that (1.3) is a sufficient closed first class constraint set, we calculate it directly from $A_{P}$ in (2.17) and $D, \bar{D}$ in (2.14), then obtain a necessary first class constraint set as

$$
\begin{aligned}
\mathcal{A}_{\mathrm{P}} & =\frac{1}{2} \operatorname{tr}\left(\langle\mathbf{D}\rangle^{2}-\langle\overline{\mathbf{D}}\rangle^{2}+2 D \bar{D}\right) \\
\mathcal{B}_{\mathrm{P} a}{ }^{\bar{b}} & =\langle\mathbf{D}\rangle_{a}{ }^{b} D_{b}{ }^{\bar{b}}+D_{a}{ }^{\bar{a}}\langle\overline{\mathbf{D}}\rangle_{\bar{a}}{ }^{\bar{b}} \\
\overline{\mathcal{B}}_{\mathrm{P} \bar{a}}{ }^{b} & =\langle\overline{\mathbf{D}}\rangle_{\bar{a}}{ }^{\bar{b}} \bar{D}_{\bar{b}}{ }^{b}+\bar{D}_{\bar{a}}{ }^{a}\langle\mathbf{D}\rangle_{a}{ }^{b} \\
c_{\mathrm{P}(a b)} & =D_{(a \mid}{ }^{\bar{a}} \bar{D}_{\bar{a} \mid b)}, \quad \bar{c}_{\mathrm{P}(\bar{a} \bar{b})}=\bar{D}_{(\bar{a} \mid}{ }^{a} D_{a \mid \bar{b})} \\
c_{\mathrm{P}\langle a b\rangle} & =D_{\langle a|}{ }^{\bar{a}} \bar{D}_{\bar{a}|b\rangle}, \quad \bar{c}_{\mathrm{P}\langle\bar{a} \bar{b}\rangle}=\bar{D}_{\langle\bar{a}|}{ }^{a} D_{a|\bar{b}\rangle} .
\end{aligned}
$$

The reparametrization constraint is $\mathcal{A}_{\mathrm{P}}$ which is the Hamiltonian (2.6) to preserve all symmetry constraints up to bi-linears of constraints. The $\kappa$-symmetry constraints are $\mathcal{B}_{\mathrm{P}}$ and $\overline{\mathcal{B}}_{\mathrm{P}}$, and others are required for closure. The first class constraint set (3.1) can be written as

$$
D_{A}^{2}{ }^{B}-\frac{1}{4+4} \delta_{A}^{B} \operatorname{Tr} D^{2}=0
$$

where $\operatorname{Tr} M=\sum M_{A}^{A}$ should not be confused with $\operatorname{STr} M=\sum(-)^{A} M^{A}{ }_{A}$. The number of constraints for $\mathcal{A}_{\mathrm{P}}, \mathcal{B}_{\mathrm{P}}, \overline{\mathcal{B}}_{\mathrm{P}}$ are $1,16,16$ respectively. Total number of constraints is $1+16 \times 2+10 \times 2+5 \times 2=63=(4+4)^{2}-1$ corresponding to the dimension of $G L(4 \mid 4) / G L(1)$. This result beginning with the AdS superparticle action (2.4) differs from (1.3) just trace part of $D^{2}$. Imposing both trace part and supertrace part of $D^{2}$ constraints means massless constraints in both $\mathrm{AdS}_{5}$ space and $\mathrm{S}^{5}$ space, which is too strong for single particle.

The constraints are reduced in a light-cone-like gauge as follows. Using with following projection operators of AdS spinors

$$
\mathcal{P}_{ \pm a}{ }^{b}=\frac{1}{2}\left(\gamma_{\mp} \gamma_{ \pm}\right)_{a}{ }^{b}, \quad \mathcal{P}_{+}=\left(\begin{array}{ll}
1 & 0 \\
0 & 0
\end{array}\right), \quad \mathcal{P}_{-}=\left(\begin{array}{ll}
0 & 0 \\
0 & 1
\end{array}\right)
$$

supercovariant derivatives are decomposed into

$$
\begin{aligned}
D_{a}{ }^{\bar{b}} & =\mathcal{P}_{+} D_{+}+\mathcal{P}_{-} D_{-} \quad, \quad \bar{D}_{\bar{a}}{ }^{b}=\bar{D}_{+} \mathcal{P}_{+}+\bar{D}_{-} \mathcal{P}_{-} \\
\langle\mathbf{D}\rangle_{a}{ }^{b} & =\mathcal{P}_{+}\langle\mathbf{D}\rangle_{+} \mathcal{P}_{-}+\mathcal{P}_{-}\langle\mathbf{D}\rangle_{-} \mathcal{P}_{+}+\mathcal{P}_{+}\langle\mathbf{D}\rangle_{\perp} \mathcal{P}_{+}+\mathcal{P}_{-}\langle\mathbf{D}\rangle_{\perp} \mathcal{P}_{-}
\end{aligned}
$$

Assuming $\mathcal{P}_{+}\langle\mathbf{D}\rangle_{+} \mathcal{P}_{-} \neq 0, \mathcal{B}_{\mathrm{P}}=0=\overline{\mathcal{B}}_{\mathrm{P}}$ are solved for $D_{-}$and $\bar{D}_{+}$. This allows the following light-cone-like gauge

$$
\begin{aligned}
& \operatorname{tr}\left[\langle\mathbf{D}\rangle_{+} \gamma_{+}\right]=4 p_{+}, \\
& \bar{\Theta}_{-\bar{m}}{ }^{a} \equiv \bar{\Theta}_{\bar{m}}{ }^{b} \mathcal{P}_{-b}{ }^{a}=0, \\
& \Theta_{+m}{ }^{\bar{c}} \equiv X_{m}{ }^{a} \mathcal{P}_{+a}{ }^{b} X^{-1}{ }_{b}{ }^{n} \Theta_{n}{ }^{\bar{c}}=0,
\end{aligned}
$$

where $X \mathcal{P}_{ \pm} X^{-1}$ matrix can be used as the projection for $\Theta_{m}{ }^{\bar{a}}$ because of invertibility of $X_{m}{ }^{a}$. The $p_{+}$is assumed not to be zero. These gauge fixing conditions (3.5) make $\mathcal{B}_{\mathrm{P}+}$ 
and $\overline{\mathcal{B}}_{\mathrm{P}-}$ to be second class and they are solved as

$$
\begin{aligned}
\bar{\zeta}_{-} & =-\frac{1}{2 p_{+}}\left[\langle\mathbf{D}\rangle_{\perp} D_{+}+D_{+}\langle\overline{\mathbf{D}}\rangle\right] \bar{X}^{-1}-\left\langle\mathbf{D}_{\perp}\right\rangle X^{-1} \Theta_{-} \bar{X}^{-1} \\
\zeta_{+} & =-\frac{1}{2 p_{+}}\left[\bar{D}_{-}\langle\mathbf{D}\rangle_{\perp}+\langle\overline{\mathbf{D}}\rangle \bar{D}_{-}\right] \gamma_{+} X^{-1}-\bar{P} \bar{\Theta}_{+} X^{-1}
\end{aligned}
$$

where $\bar{\zeta}_{-}$and $\zeta_{+}$are conjugates of $\bar{\Theta}_{-}$and $\Theta_{+}$defined through $\mathcal{P}_{-} \bar{\zeta}$ and $\zeta X \mathcal{P}_{+} X^{-1}$ respectively. $\mathcal{A}_{\mathrm{P}}=0$ can be written as

$$
\operatorname{tr}\left[\langle\mathbf{D}\rangle_{-} \gamma_{-}\right]=\frac{-1}{2 p_{+}} \operatorname{tr}\left[\langle\mathbf{D}\rangle_{\perp}^{2}-\langle\overline{\mathbf{D}}\rangle^{2}\right]+\frac{1}{p_{+}{ }^{2}} \operatorname{tr}\left[\langle\mathbf{D}\rangle_{\perp} \gamma_{+} D_{+} \bar{D}_{-}-\langle\overline{\mathbf{D}}\rangle \overline{D_{-}} \gamma_{+} D_{+}\right]
$$

Remaining components of constraints $\mathcal{B}_{\mathrm{P}-}, \overline{\mathcal{B}}_{\mathrm{P}+}, c_{P}$ and $\bar{c}_{\mathrm{P}}$ are first class constraints written by $D_{+}, \bar{D}_{-}, p_{+},\langle\mathbf{D}\rangle_{\perp}$ and $\langle\overline{\mathbf{D}}\rangle$

$$
\begin{aligned}
\mathcal{B}_{\mathrm{P}-} & =\frac{1}{4 p_{+}^{2}} \gamma_{+} D_{+} \operatorname{tr}\left[c_{\mathrm{P} a_{+} b_{-}}\langle\mathbf{D}\rangle_{\perp} \gamma_{+}-\langle\overline{\mathbf{D}}\rangle \bar{D}_{-} \gamma_{+} D_{+}\right]=0 \\
\overline{\mathcal{B}}_{\mathrm{P}+} & =\frac{1}{4 p_{+}^{2}} \bar{D}_{-} \gamma_{+} \operatorname{tr}\left[c_{\mathrm{P} a_{+} b_{-}}\langle\mathbf{D}\rangle_{\perp} \gamma_{+}-\langle\overline{\mathbf{D}}\rangle \bar{D}_{-} \gamma_{+} D_{+}\right]=0 \\
c_{\mathrm{P}} & =D_{+} \bar{D}_{-}, \\
\bar{c}_{\mathrm{P}} & =-\frac{1}{p_{+}}\langle\overline{\mathbf{D}}\rangle \bar{D}_{-} \gamma_{+} D_{+} .
\end{aligned}
$$

It is noted that $\mathcal{B}_{\mathrm{P}}$ and $\overline{\mathcal{B}}_{\mathrm{P}}$ are fixed up to a square of fermionic constraints which can be set to zero in the second class approach, so $\mathcal{B}_{\mathrm{P}-}$ and $\overline{\mathcal{B}}_{\mathrm{P}+}$ do not produce further constraints. But this is not the case in the first class approach. We take a sufficient closed constraint set to examine the restricted states,

$$
c_{\mathrm{P}} \rightarrow D_{+} \bar{D}_{-}=0, \quad \mathcal{B}, \overline{\mathcal{B}}, \bar{c}_{\mathrm{P}} \rightarrow \bar{D}_{-} \gamma_{+} D_{+}=0
$$

Independent supercovariant derivatives $D_{+}$and $\bar{D}_{-}$satisfy

$$
\left[\left(D_{+}\right)_{a}{ }^{\bar{b}},\left(\bar{D}_{-}\right)_{\bar{c}}{ }^{d}\right]=-p_{+} \delta_{\bar{c}}^{\bar{b}}\left(\gamma_{-}\right)_{a}{ }^{d} .
$$

Independent fermionic degrees of freedom are $8 Q_{+}$and $8 \bar{Q}_{-}$

$$
\begin{array}{lll}
Q_{+}=X \mathcal{P}_{+} X^{-1} Q & , & Q_{m}{ }^{\bar{n}}=X \bar{\zeta}+\Theta \bar{P} \\
\bar{Q}_{-}=\bar{Q} X \mathcal{P}_{-} X^{-1} & , & \bar{Q}_{\bar{m}}{ }^{n}=\bar{X} \zeta+\bar{\Theta} P
\end{array}
$$

which satisfy (1.1) and anticommute with $D_{+}$and $\bar{D}_{-}$. The poisson bracket (3.9) are rewritten as the four sets of 4-dimensional Clifford algebras, and further can be written by two sets of 4-dimensional creation-annihilation commutators in $\mathrm{U}(2) \times \mathrm{U}(4)$ basis as

$$
\left[\overline{\mathbf{d}}_{i}^{\bar{a}}, \mathbf{d}_{j}^{\bar{b}}\right]=\delta_{i j} \delta^{\bar{a} \bar{b}}
$$

In (3.8) $D_{+} \bar{D}_{-}$'s are generators of $\mathrm{GL}(2) \cong \mathrm{U}(2)$ and $\bar{D}_{-} \gamma_{+} D_{+}$'s are generators of $\mathrm{GL}(4) \cong \mathrm{U}(4)$, which are imposed as

$$
\langle\mathbf{d}|V| \mathbf{d}\rangle=0 \quad, \quad V=\left\{D_{+} \bar{D}_{-}, \quad \bar{D}_{-} \gamma_{+} D_{+}\right\}
$$


where

$$
|\mathbf{d}\rangle=\left\{|\phi\rangle, \mathbf{d}|\phi\rangle, \cdots, \mathbf{d}^{8}|\phi\rangle\right\}
$$

with the ground state defined by $\overline{\mathbf{d}}|\phi\rangle=0$. The conditions (3.12) is realized on the states $|\mathbf{d}\rangle$ as

$$
\begin{aligned}
& \left(V_{0}-v_{0}\right)|\mathbf{d}\rangle=0, V_{-}|\mathbf{d}\rangle=0 \\
& V_{0}=\left\{V_{0 i}=\left.\mathbf{d}_{i}^{\bar{a}} \overline{\mathbf{d}}_{i}^{\bar{a}}\right|_{\bar{a}: \text { sum }, i \text { :non-sum }}, \quad V_{0}^{\bar{a}}=\left.\mathbf{d}_{i}^{\bar{a}} \overline{\mathbf{d}}_{i}^{\bar{a}}\right|_{\bar{a}: \text { non-sum }, i: \text { sum }}\right\} \\
& V_{-}=\left\{V_{-i j}=\left.\mathbf{d}_{i}^{\bar{a}} \overline{\mathbf{d}}_{j}^{\bar{a}}\right|_{\bar{a}: \text { sum }, i>j}, \quad V_{-}^{\bar{a} \bar{b}}=\left.\mathbf{d}_{i}^{\bar{a}} \overline{\mathbf{d}}_{i}^{\bar{b}}\right|_{\bar{a}>\bar{b}, i: \text { sum }}\right\},
\end{aligned}
$$

and restricted states are $|\phi\rangle$ for $v_{0}=0$ or $\mathbf{d}_{1}^{\overline{1}}|\phi\rangle$ for $\left(v_{0}\right)_{1}=\left(v_{0}\right)^{\overline{1}}=1$ as highest weight states depending on the $v_{0}$. These are the same higest weight states with the one for a flat case. It can be obtained by imposing $\mathrm{GL}(2) \times \mathrm{GL}(4)$ invariance while it is obtained by $\mathrm{SO}(8)$ invariance for a flat case. In other words this closed first class constraint set $\left(D^{2}\right)_{A}^{B}=0$ is conformal extension of the one for a flat superparticle [14].

\section{$4 \quad$ Classical AdS superstring mechanics}

We begin with the action for a superstring given by [6]

$$
S=\int d \sigma^{2} \frac{1}{2}\left\{-\sqrt{-g} g^{\mu \nu}\left(J_{\mu}^{\langle a b\rangle} J_{\langle a b\rangle \nu}-J_{\mu}^{\langle\bar{a} \bar{b}\rangle} J_{\nu\langle\bar{a} \bar{b}\rangle}\right)+\frac{k}{2} \epsilon^{\mu \nu}\left(E^{1 / 2} J_{\mu}^{a \bar{b}} J_{\nu a \bar{b}}-E^{-1 / 2} J_{\mu}^{\bar{a} b} J_{\nu \bar{a} b}\right)\right\}
$$

with $k= \pm 1$. Canonical conjugates $P_{A}{ }^{M}$ are defined in (2.5), and we also introduce useful variables

$$
\begin{aligned}
\tilde{P}_{a}^{m} & =P_{a}^{m}+\frac{k}{2} E^{-1 / 2}\left(\bar{X}^{-1} \bar{v} \bar{\Theta} X^{-1}\right)^{\bar{b} m}\left(\bar{J}_{1}\right)_{\bar{b} a}=-\sqrt{-g}\left(g^{00} \mathbf{J}_{0}+g^{01} \mathbf{J}_{1}\right)_{a}{ }^{b}\left(X^{-1} v\right)_{b}{ }^{m} \\
\tilde{\bar{P}}_{\bar{a}}{ }^{\bar{m}} & =\bar{P}_{\bar{a}}{ }^{\bar{m}}+\frac{k}{2} E^{1 / 2}\left(X^{-1} v \Theta \bar{X}^{-1}\right)^{b \bar{m}}\left(J_{1}\right)_{b \bar{a}}=-\sqrt{-g}\left(g^{00} \overline{\mathbf{J}}_{0}+g^{01} \overline{\mathbf{J}}_{1}\right)_{\bar{a}}{ }^{\bar{b}}\left(\bar{X}^{-1} \bar{v}\right)_{\bar{b}} \bar{m} \\
\zeta_{\bar{a}}{ }^{m} & =-\left(\bar{P} \bar{\Theta} X^{-1}\right)_{\bar{a}}{ }^{m}+\frac{k}{2} E^{1 / 2}\left(X^{-1}\right)^{b m}\left(J_{1}\right)_{b \bar{a}} \\
\bar{\zeta}_{a}{ }^{\bar{m}} & =-\left(P \Theta \bar{X}^{-1}\right)_{a}{ }^{\bar{m}}+\frac{k}{2} E^{-1 / 2}\left(\bar{X}^{-1}\right)^{\bar{b} \bar{m}}\left(\bar{J}_{1}\right)_{\bar{b} a} .
\end{aligned}
$$

The last two fermionic equations are primary constraints corresponding to the one for the AdS superparticle (2.14),

$$
\begin{aligned}
& F_{a}{ }^{\bar{b}}=E^{1 / 4} D_{a}{ }^{\bar{b}}+\frac{k}{2} E^{-1 / 4}\left(\bar{J}_{1}\right)^{\bar{b}}{ }_{a}=0 \\
& \bar{F}_{\bar{a}}{ }^{b}=E^{-1 / 4} \bar{D}_{\bar{a}}{ }^{b}+\frac{k}{2} E^{1 / 4}\left(J_{1}\right)^{b}{ }_{\bar{a}}=0 .
\end{aligned}
$$


The Hamiltonian is given by

$$
\begin{aligned}
\mathcal{H} & =-\frac{2}{\sqrt{-g} g^{00}} A_{\perp}-\frac{2 g^{01}}{g^{00}} A_{\|}+\operatorname{tr}[F \bar{\lambda}+\bar{F} \lambda] \\
A_{\perp} & =\frac{1}{2} \operatorname{tr}\left[\langle\mathbf{D}\rangle^{2}+\langle\mathbf{J}\rangle^{2}-\langle\overline{\mathbf{D}}\rangle^{2}-\langle\overline{\mathbf{J}}\rangle^{2}\right]=0 \\
A_{\|} & =\operatorname{tr}[\langle\mathbf{D}\rangle\langle\mathbf{J}\rangle-\langle\overline{\mathbf{D}}\rangle\langle\overline{\mathbf{J}}\rangle]=0
\end{aligned}
$$

with multipliers $\lambda$ 's determined consistently

$$
\begin{aligned}
& \lambda=-\frac{2}{\sqrt{-g} g^{00}}\left(E^{-1 / 4} \bar{J}\right)-\frac{2 g^{01}}{g^{00}}\left(-E^{1 / 4} J\right) \\
& \bar{\lambda}=-\frac{2}{\sqrt{-g} g^{00}}\left(-E^{1 / 4} J\right)-\frac{2 g^{01}}{g^{00}}\left(E^{-1 / 4} \bar{J}\right),
\end{aligned}
$$

where we denote $J_{1}$ as $J$ from now on.

The poisson brackets of the fermionic constraints (4.3) are given schematically as

$$
\begin{aligned}
{\left[\left(\begin{array}{c}
F_{a}{ }^{\bar{b}} \\
\bar{F}_{\bar{a}}{ }^{b}
\end{array}\right),\left(F_{c}{ }^{\bar{d}}, \bar{F}_{\bar{c}}{ }^{d}\right)\right] } & =\left(\begin{array}{cc}
J_{a c}^{\bar{b} \bar{d}} & D_{a \bar{c}}^{d \bar{b}} \\
D_{\bar{a} c}^{b \bar{d}} & J_{\bar{a} \bar{c}}^{b d}
\end{array}\right) \\
J_{a c}^{\bar{b} \bar{d}} & =\mathbf{J}_{\langle a c\rangle} \Omega^{\bar{b} \bar{d}}-\overline{\mathbf{J}}^{\langle\bar{b} \bar{d}\rangle} \Omega_{a c}-\frac{1}{4} \operatorname{STr} J \Omega_{a c} \Omega^{\bar{b} \bar{d}} \\
J_{\bar{a} \bar{c}}^{b d} & =\mathbf{J}^{\langle b d\rangle} \Omega_{\bar{a} \bar{c}}-\overline{\mathbf{J}}_{\langle\bar{a} \bar{c}\rangle} \Omega^{b d}-\frac{1}{4} \operatorname{STr} J \Omega_{\bar{a} \bar{c}} \Omega^{b d} \\
D_{a \bar{c}}^{d \bar{b}} & =-\mathbf{D}_{a}{ }^{d} \delta_{\bar{c}} \delta_{\bar{b}} \overline{\mathbf{D}}_{\bar{c}}{ }^{\bar{b}} \delta_{a}^{d} \\
D_{\bar{a} c}^{b \bar{d}} & =-\mathbf{D}_{c}{ }^{b} \delta_{\bar{a}} \delta_{\bar{d}}+\overline{\mathbf{D}}_{\bar{a}} \bar{d}_{c} \delta_{c}^{b} .
\end{aligned}
$$

The right hand side of (4.8) has half zero modes described symbolically as

$$
\left(\begin{array}{cc}
D & J \\
J & D
\end{array}\right)\left(\begin{array}{cc}
J & D \\
D & J
\end{array}\right)=\left(\begin{array}{cc}
A_{\|} & A_{\perp} \\
A_{\perp} & A_{\|}
\end{array}\right)=0
$$

by using the $\tau$ and $\sigma$ reparametrization constraints (4.5) and (4.6). As expected the half of the fermionic constraints are first class and another half are second class constraints. The projection matrix into first class is now the one in (4.9), so first class constraints are given by

$$
\left(\begin{array}{c}
B \\
\bar{B}
\end{array}\right) \equiv\left(\begin{array}{cc}
D & J \\
J & D
\end{array}\right)\left(\begin{array}{c}
F \\
\bar{F}
\end{array}\right)=\left(\begin{array}{c}
\langle\mathbf{D}\rangle F+F\langle\overline{\mathbf{D}}\rangle-(\langle\overline{\mathbf{J}}\rangle \bar{F}+\bar{F}\langle\mathbf{J}\rangle)^{T} \\
\langle\overline{\mathbf{D}}\rangle \bar{F}+\bar{F}\langle\mathbf{D}\rangle-(\langle\mathbf{J}\rangle F+F\langle\overline{\mathbf{J}}\rangle)^{T}
\end{array}\right)=0
$$

They generate following $\kappa$-transformations

$$
\begin{aligned}
\delta_{\kappa, \bar{\kappa}} Z & =[Z, \operatorname{tr}(\bar{B} \kappa-B \bar{\kappa})] \\
\delta_{\kappa, \bar{\kappa}} X & =\Theta\left(\bar{\kappa}\langle\mathbf{D}\rangle+\langle\overline{\mathbf{D}}\rangle \bar{\kappa}+\kappa^{T}\langle\mathbf{J}\rangle+\langle\overline{\mathbf{J}}\rangle \kappa^{T}\right) \\
\delta_{\kappa, \bar{\kappa}} \bar{X} & =\bar{\Theta}\left(\kappa\langle\overline{\mathbf{D}}\rangle+\langle\mathbf{D}\rangle \kappa+\bar{\kappa}^{T}\langle\overline{\mathbf{J}}\rangle+\langle\mathbf{J}\rangle \bar{\kappa}^{T}\right) \\
\delta_{\kappa, \bar{\kappa}} \Theta & =X\left(\kappa\langle\overline{\mathbf{D}}\rangle+\langle\mathbf{D}\rangle \kappa+\bar{\kappa}^{T}\langle\overline{\mathbf{J}}\rangle+\langle\mathbf{J}\rangle \bar{\kappa}^{T}\right) \\
\delta_{\kappa, \bar{\kappa}} \bar{\Theta} & =\bar{X}\left(\bar{\kappa}\langle\mathbf{D}\rangle+\langle\overline{\mathbf{D}}\rangle \bar{\kappa}+\kappa^{T}\langle\mathbf{J}\rangle+\langle\overline{\mathbf{J}}\rangle \kappa^{T}\right) .
\end{aligned}
$$


Both $\kappa$ and $\bar{\kappa}$ parameters have following zeromodes, and this reducibility continues infinitely

$$
\begin{aligned}
& \delta \kappa=\langle\mathbf{D}\rangle \kappa_{1}-\kappa_{1}\langle\overline{\mathbf{D}}\rangle-\langle\mathbf{J}\rangle \bar{\kappa}_{1}^{T}+\bar{\kappa}_{1}^{T}\langle\overline{\mathbf{J}}\rangle, \delta \kappa_{1}=\langle\mathbf{D}\rangle \kappa_{2}+\kappa_{2}\langle\overline{\mathbf{D}}\rangle+\langle\mathbf{J}\rangle \bar{\kappa}_{2}^{T}+\bar{\kappa}_{2}^{T}\left\langle\overline{\mathbf{J}}^{T}\right\rangle, \cdots \\
& \delta \bar{\kappa}=\langle\overline{\mathbf{D}}\rangle \bar{\kappa}_{1}-\bar{\kappa}_{1}\langle\mathbf{D}\rangle-\langle\overline{\mathbf{J}}\rangle \kappa_{1}^{T}+\kappa_{1}^{T}\langle\mathbf{J}\rangle, \delta \bar{\kappa}_{1}=\langle\overline{\mathbf{D}}\rangle \bar{\kappa}_{2}+\bar{\kappa}_{2}\langle\mathbf{D}\rangle+\langle\overline{\mathbf{J}}\rangle \kappa_{2}^{T}+\kappa_{2}^{T}\langle\mathbf{J}\rangle, \cdots
\end{aligned}
$$

where $A_{\perp}=0$ and $A_{\|}=0$ are used. Again it leads to that only each half components of $\Theta$ and $\bar{\Theta}$ can be fixed by using this $\kappa$-symmetries.

Now we calculate equations of motion using with the Hamiltonian (4.4) for an AdS superstring in the conformal gauge $g_{00}+g_{11}=0=g_{01}$,

$$
\partial_{\tau} Z_{M}{ }^{A}=Z_{M}{ }^{B} \Gamma_{B}{ }^{A} \quad, \quad \Gamma=\left(\begin{array}{cc}
\langle\mathbf{D}\rangle & -E^{-1 / 2} \bar{J}^{T} \\
-E^{1 / 2} J^{T} & \langle\overline{\mathbf{D}}\rangle
\end{array}\right) \approx\left(\begin{array}{cc}
\langle\mathbf{D}\rangle & 2 D \\
2 \bar{D} & \langle\overline{\mathbf{D}}\rangle
\end{array}\right) .
$$

The momenta satisfies

$$
\begin{array}{ll}
\partial_{\tau}\langle\mathbf{D}\rangle=\nabla_{\sigma}\langle\mathbf{J}\rangle, & \partial_{\tau}\langle\mathbf{J}\rangle=\nabla_{\sigma}\langle\mathbf{D}\rangle \\
\partial_{\tau}\langle\overline{\mathbf{D}}\rangle=\nabla_{\sigma}\langle\overline{\mathbf{J}}\rangle, & \partial_{\tau}\langle\overline{\mathbf{J}}\rangle=\nabla_{\sigma}\langle\overline{\mathbf{D}}\rangle
\end{array},
$$

which is the stringy extension of momentum conservation of the superparticle, $\langle\dot{\mathbf{D}}\rangle=0$. The field equation is given by

$$
\begin{aligned}
& \left\{-\partial_{\tau}^{2}+\partial_{\sigma}^{2}+\frac{1}{2} \hat{\Gamma}_{+} \hat{\Gamma}_{-}+\frac{1}{2} \hat{\Gamma}_{-} \hat{\Gamma}_{+}\right. \\
& \left.+\left[\left.\hat{\Gamma}_{+}\right|_{\text {bose }},\left.\hat{\Gamma}_{-}\right|_{\text {fermi }}\right]+\left[\left.\hat{\Gamma}_{-}\right|_{\text {bose }},\left.\hat{\Gamma}_{+}\right|_{\text {fermi }}\right]\right\}_{B}^{A} Z_{M}^{B}=0, \\
& \hat{\Gamma}_{ \pm}=\left(\begin{array}{cc}
\left\langle\hat{\mathbf{D}}_{ \pm}\right\rangle & 2 \hat{\hat{D}}_{ \pm} \\
2 \hat{\bar{D}}_{ \pm} & \left\langle\hat{\overline{\mathbf{D}}}_{ \pm}\right\rangle
\end{array}\right) \\
& \langle\hat{\mathbf{D}}\rangle_{ \pm}=\langle\mathbf{D}\rangle \pm\langle\mathbf{J}\rangle,\langle\hat{\overline{\mathbf{D}}}\rangle_{ \pm}=\langle\overline{\mathbf{D}}\rangle \pm\langle\overline{\mathbf{J}}\rangle \\
& \hat{\hat{D}}_{ \pm}=D \pm \frac{1}{2} J \quad, \quad \hat{\bar{D}}_{ \pm}=\bar{D} \pm \frac{1}{2} \bar{J}
\end{aligned}
$$

in a trivial $S p(4) \times G L(1)$ gauge. $\hat{\Gamma}_{ \pm}$are now induced connection of AdS background on the right/left sectors of the worldvolume.

We have shown that the AdS superstring has the $\tau, \sigma$ reparametrization invariance and the fermionic constraints that are mixture of first class and second class as same as the flat superstring. The field equation for the AdS superstring is given by (4.15) which includes the contribution of the AdS background through the induced connections $\hat{\Gamma}$ 's.

It is expected that the solutions are written as sum of right and left moving modes. Indeed the matrix in the right hand side of (4.8) can be diagonalized, where eigenvalues are

$$
\begin{aligned}
& \hat{\mathbf{D}}_{ \pm}=\mathbf{D} \pm \mathbf{J} \\
& \hat{\mathbf{D}}_{ \pm}=\overline{\mathbf{D}} \pm \overline{\mathbf{J}}
\end{aligned}
$$


and eigenstates are

$$
\hat{D}_{ \pm a}^{\bar{b}}=F_{a}^{\bar{b}} \pm \bar{F}_{a}^{\bar{b}}=E^{1 / 4} \hat{\hat{D}}_{a}^{\bar{b}} \pm E^{-1 / 4} \hat{\bar{D}}_{a}^{\bar{b}}
$$

The coset part of supercovariant derivative can be separated into right/left moving sectors up to constraints

$$
\begin{aligned}
{\left[\left\langle\hat{\mathbf{D}}_{ \pm}\right\rangle_{a b}(\sigma),\left\langle\hat{\mathbf{D}}_{\mp}\right\rangle_{c d}\left(\sigma^{\prime}\right)\right] } & =2 \Omega_{\langle c|\langle b}(\mathbf{D})_{a\rangle|d\rangle} \approx 0 \\
{\left[\left\langle\hat{\mathbf{D}}_{ \pm}\right\rangle_{a b}(\sigma), \hat{D}_{\mp, c}^{\bar{d}}\left(\sigma^{\prime}\right)\right] } & =\Omega_{c\langle b} \hat{D}_{ \pm, a\rangle}^{\bar{d}} \delta\left(\sigma-\sigma^{\prime}\right) \approx 0 \\
{\left[\hat{D}_{ \pm, a}^{\bar{b}}(\sigma), \hat{D}_{\mp, c}^{\bar{d}}\left(\sigma^{\prime}\right)\right] } & =2\left(\Omega_{a c}(\overline{\mathbf{D}})^{\bar{b} \bar{d}}+\Omega^{\bar{b}} \bar{d}(\mathbf{D})_{a c}\right) \approx 0
\end{aligned}
$$

and similar algebras for the bared $\left(\mathrm{S}^{5}\right)$ sector. This right/left separation corresponds to the choice $J_{\text {bose }} \rightarrow R J_{\text {bose }}$ and $J_{\text {fermi }} \rightarrow(R / 2) J_{\text {fermi }}$ in the IW contraction (1.9). Despite of the general feature of the algebra (1.7), this almost chiral representation is possible because of local constraints such as bosonic $\operatorname{Sp}(4)$ constraints $\mathbf{D}_{(a b)}=\overline{\mathbf{D}}_{(a b)}=0$. On the other hand this incomplete separation causes the following complicated algebra. The poisson bracket of supercovariant derivatives for a $\tau+\sigma$ sector are given as follows, where + indices are omitted:

$$
\begin{aligned}
{\left[\langle\hat{\mathbf{D}}\rangle_{a b}(\sigma),\langle\hat{\mathbf{D}}\rangle_{c d}\left(\sigma^{\prime}\right)\right] } & =2 \Omega_{\langle c|\langle b} \Omega_{a\rangle|d\rangle} \delta^{\prime}\left(\sigma-\sigma^{\prime}\right)+4 \Omega_{\langle c|\langle b}(\mathbf{J})_{a\rangle|d\rangle} \delta\left(\sigma-\sigma^{\prime}\right) \\
{\left[\langle\hat{\overline{\mathbf{D}}}\rangle_{\bar{a} \bar{b}}(\sigma),\langle\hat{\overline{\mathbf{D}}}\rangle_{\bar{c} \bar{d}}\left(\sigma^{\prime}\right)\right] } & =-2 \Omega_{\langle\bar{c}|\langle\bar{b}} \Omega_{\bar{a}\rangle|\bar{d}\rangle} \delta^{\prime}\left(\sigma-\sigma^{\prime}\right)+4 \Omega_{\langle\bar{c}|\langle\bar{b}}(\overline{\mathbf{J}})_{\bar{a}\rangle|\bar{d}\rangle} \delta\left(\sigma-\sigma^{\prime}\right) \\
{\left[\hat{D}_{a}{ }^{\bar{b}}(\sigma), \hat{D}_{c}{ }^{\bar{d}}\left(\sigma^{\prime}\right)\right] } & =2\left(\Omega^{\bar{b}} \bar{d}\langle\hat{\mathbf{D}}\rangle_{a c}-\Omega_{a c}\langle\hat{\overline{\mathbf{D}}}\rangle^{\bar{b} \bar{d}}\right) \delta\left(\sigma-\sigma^{\prime}\right) \\
{\left[\langle\hat{\mathbf{D}}\rangle_{a b}(\sigma), \hat{D}_{c}^{\bar{d}}\left(\sigma^{\prime}\right)\right] } & =\Omega_{c\langle b} \tilde{D}_{a\rangle}^{\bar{d}} \delta\left(\sigma-\sigma^{\prime}\right) \\
{\left[\langle\hat{\overline{\mathbf{D}}}\rangle_{\bar{a} \bar{b}}(\sigma), \hat{D}_{c}^{\bar{d}}\left(\sigma^{\prime}\right)\right] } & =\delta_{\langle\bar{a}}^{\bar{d}} \tilde{D}_{c|\bar{b}\rangle} \delta\left(\sigma-\sigma^{\prime}\right) \\
{\left[\tilde{D}_{a}{ }^{\bar{b}}(\sigma), \tilde{D}_{c}{ }^{\bar{d}}\left(\sigma^{\prime}\right)\right] } & =2\left(-\Omega^{\bar{b} \bar{d}}\langle\hat{\mathbf{D}}+2 \mathbf{J}\rangle^{a c}+\Omega_{a c}\langle\hat{\overline{\mathbf{D}}}+2 \overline{\mathbf{J}}\rangle^{\bar{b} \bar{d}}\right) \delta\left(\sigma-\sigma^{\prime}\right) \\
{\left[\hat{D}_{a}{ }^{\bar{b}}(\sigma), \tilde{D}_{c}{ }^{\bar{d}}\left(\sigma^{\prime}\right)\right] } & \left.=-4 \Omega_{a c} \Omega^{\bar{b} \bar{d}} \delta^{\prime}\left(\sigma-\sigma^{\prime}\right)-4\left\{\Omega_{a c}(\overline{\mathbf{J}})^{\bar{b} \bar{d}}+\Omega^{\bar{b} \bar{d}}(\mathbf{J})_{a c}\right)\right\} \delta\left(\sigma-\sigma^{\prime}\right) \\
{\left[\langle\hat{\mathbf{D}}\rangle_{a b}(\sigma), \tilde{D}_{c}{ }^{\bar{d}}\left(\sigma^{\prime}\right)\right] } & =\Omega_{c\langle b}(\hat{D}-2 \hat{\omega})_{a\rangle} \bar{d} \delta\left(\sigma-\sigma^{\prime}\right) \\
{\left[\langle\hat{\overline{\mathbf{D}}}\rangle_{\bar{a} \bar{b}}(\sigma), \tilde{D}_{c}{ }^{\bar{d}}\left(\sigma^{\prime}\right)\right] } & =\delta_{\langle\bar{a}}^{\bar{d}}(\hat{D}-2 \hat{\omega})_{c|\bar{b}\rangle} \delta\left(\sigma-\sigma^{\prime}\right)
\end{aligned}
$$

where

$$
\begin{aligned}
\tilde{D}_{ \pm a}^{\bar{b}} & =\hat{D}_{\mp a}^{\bar{b}}+2 \hat{\omega}_{ \pm a}^{\bar{b}} \\
\hat{\omega}_{ \pm a}^{\bar{b}} & = \pm E^{1 / 4} J_{a}^{\bar{b}}-E^{-1 / 4} \bar{J}_{a}^{\bar{b}} .
\end{aligned}
$$

Then next we calculate closed first class constraint set to obtain its local symmetries analogous to the AdS superparticle case. The first class constraint set, which can be separated into the left/right chiral sectors up to the constraints, is given by

$$
\mathcal{A}=\frac{1}{4} \operatorname{tr}\left(\langle\hat{\mathbf{D}}\rangle^{2}-\langle\hat{\overline{\mathbf{D}}}\rangle^{2}+\tilde{D} \hat{D}\right)
$$




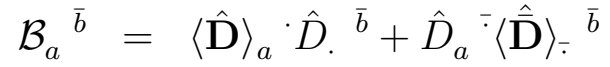

$$
\begin{aligned}
& \mathcal{C}_{a c}^{\bar{b} \bar{d}}=\hat{D}_{a}^{\bar{b}} \hat{D}_{c}^{\bar{d}} \\
& \mathcal{D}_{\langle a b\rangle}=\hat{D}_{\langle a}{ }^{\bar{D}} \nabla \hat{D}_{b\rangle^{-}}, \quad \overline{\mathcal{D}}_{\langle\bar{a} \bar{b}\rangle}=\hat{D}_{\langle\bar{a}|} \nabla \hat{D}_{\cdot|\bar{b}\rangle},
\end{aligned}
$$

where indices $\cdot, \overline{-}$ are used as contracted indices. The number of $\mathcal{A}, \mathcal{B}, \mathcal{C}, \mathcal{D}$ and $\overline{\mathcal{D}}$ are $1,16,16 \times 15 / 2=120,5$ and 5 which coincides with the flat superstring case [0]. They satisfy the following algebras up to (2.13) :

$$
\begin{aligned}
& {\left[\mathcal{A}(\sigma), \mathcal{A}\left(\sigma^{\prime}\right)\right]=2 \mathcal{A}(\sigma) \delta^{\prime}\left(\sigma-\sigma^{\prime}\right)+\mathcal{A}^{\prime} \delta\left(\sigma-\sigma^{\prime}\right)} \\
& {\left[\mathcal{A}(\sigma), \mathcal{B}_{c}{ }^{\bar{d}}\left(\sigma^{\prime}\right)\right]=-2 \mathcal{B}_{c}{ }^{\bar{d}} \delta^{\prime}\left(\sigma-\sigma^{\prime}\right)+\left\{-\nabla \mathcal{B}_{c}{ }^{\bar{d}}+\frac{1}{2} \mathcal{C}_{c}{ }^{\overline{ }}\left\langle\hat{\omega}_{-}{ }^{\bar{d}}\right\rangle-\frac{1}{2}\left\langle\hat{\omega}_{-}{ }_{c}{ }_{c} \mathcal{C} \cdot{ }_{-}\right\rangle .{ }^{\bar{d}}\right\} \delta\left(\sigma-\sigma^{\prime}\right)} \\
& \nabla \mathcal{B}_{c}{ }^{\bar{d}}=\left(\mathcal{B}^{\prime}-2(\mathbf{J}) \mathcal{B}+2 \mathcal{B}(\overline{\mathbf{J}})\right)_{c}{ }^{\bar{d}} \\
& {\left[\mathcal{A}(\sigma), \mathcal{C}_{a c}^{\bar{d}} \bar{d}\left(\sigma^{\prime}\right)\right]=2 \mathcal{C}_{a c}^{\bar{b}} \bar{d}(\sigma) \delta^{\prime}\left(\sigma-\sigma^{\prime}\right)+\mathcal{C}_{a c}^{\prime} \bar{b} \bar{d}(\sigma) \delta\left(\sigma-\sigma^{\prime}\right)} \\
& {\left[\mathcal{A}(\sigma), \mathcal{D}_{a b}\left(\sigma^{\prime}\right)\right]=-3 \mathcal{D}_{a b}(\sigma) \delta^{\prime}\left(\sigma-\sigma^{\prime}\right)-2 \mathcal{D}_{a b}^{\prime}(\sigma) \delta\left(\sigma-\sigma^{\prime}\right)} \\
& {\left[\mathcal{A}(\sigma), \overline{\mathcal{D}}_{\bar{a} \bar{b}}\left(\sigma^{\prime}\right)\right]=-3 \overline{\mathcal{D}}_{\bar{a} \bar{b}}(\sigma) \delta^{\prime}\left(\sigma-\sigma^{\prime}\right)-2 \overline{\mathcal{D}}_{\bar{a} \bar{b}}^{\prime}(\sigma) \delta\left(\sigma-\sigma^{\prime}\right)} \\
& {\left[\mathcal{B}_{a}{ }^{\bar{b}}(\sigma), \mathcal{B}_{c}{ }^{\bar{d}}\left(\sigma^{\prime}\right)\right]=-\left(\mathcal{C}_{\langle a c\rangle^{\cdot}}^{\bar{*}}+\mathcal{C}^{\cdot\langle\bar{b}}{ }^{\bar{d}\rangle}+2 \mathcal{C}_{a c}^{\bar{d}} \bar{b}\right) \delta^{\prime}\left(\sigma-\sigma^{\prime}\right)} \\
& -2\left[\left(\langle\hat{\mathbf{D}}\rangle_{a c} \Omega^{\bar{b} \bar{d}}+\langle\hat{\overline{\mathbf{D}}}\rangle^{\bar{b} \bar{d}} \Omega_{a c}\right) \mathcal{A}\right. \\
& +\frac{1}{2}\left(\mathcal{B}_{a}{ }^{\bar{b}} \tilde{D}_{c}{ }^{\bar{d}}+\frac{a}{b} \leftrightarrow \frac{c}{\bar{d}}\right)+\left(\mathcal{B} \tilde{D}^{T}\right)_{\langle a c\rangle} \Omega^{\bar{b} \bar{d}}-\left(\tilde{D}^{T} \mathcal{B}\right)^{\langle\bar{b} \bar{d}\rangle} \Omega_{a c} \\
& \left.-\nabla \mathcal{C}_{a c}{ }_{a}^{\bar{d}} \bar{b}+\mathcal{D}_{a c} \Omega^{\bar{b} \bar{d}}-\overline{\mathcal{D}}^{\bar{b}} \bar{d} \Omega_{a c}\right] \delta\left(\sigma-\sigma^{\prime}\right) \\
& \nabla \mathcal{C}_{a c}^{\bar{d} \bar{b}}=\mathcal{C}_{a c}^{\prime} \bar{d} \bar{b}+\mathcal{C}_{*}{ }_{*}^{\bar{*}}\left[\left((\mathbf{J})_{a} \delta_{\bar{*}}^{\bar{d}}+(\overline{\mathbf{J}})_{:^{d}}^{\bar{d}} \delta_{a}^{*}\right) \delta_{c}^{*} \delta_{\bar{*}}^{\bar{b}}+_{a}^{\bar{d}} \leftrightarrow_{c}^{\bar{b}}\right) \\
& \left.-\Omega_{a c}(\mathbf{J})^{* *} \delta^{[\bar{b}} \delta_{\bar{*}}^{\bar{d}]}+\Omega^{\bar{b} \bar{d}}(\overline{\mathbf{J}})_{\bar{*}^{*}} \delta_{[a} \delta_{c]}^{*}+(\mathbf{J})_{a c} \delta_{\cdot}^{(\bar{b}} \delta_{\bar{*}}^{\bar{d})} \Omega^{* \cdot}-(\overline{\mathbf{J}})^{\bar{b} \bar{d}} \delta_{(a} \delta_{c}^{*} \Omega_{\bar{*} \cdot]}\right] \\
& {\left[\mathcal{B}_{a}{ }^{\bar{b}}(\sigma), \mathcal{C}_{c d}^{\bar{c} d} \bar{d}\left(\sigma^{\prime}\right)\right]=\left[-2 \Omega_{a c} \Omega^{\bar{b} \bar{c}} \hat{D}_{d}^{\bar{d}} \mathcal{A}+\frac{1}{2}\left\{\left(\delta_{c} \delta_{*}^{\bar{b}} \delta_{d}^{*} \delta_{\bar{k}}^{\bar{d}} \tilde{D}_{a}^{\bar{c}}-{ }_{a}^{\bar{c}} \leftrightarrow_{c}^{\bar{b}}\right)-\left(\delta_{d} \delta_{*}^{\bar{b}} \delta_{c}^{*} \delta_{\bar{*}}^{\bar{c}} \tilde{D}_{a}{ }_{a}^{\bar{d}}-{ }_{a}^{\bar{d}} \leftrightarrow{ }_{d}^{\bar{b}}\right)\right.\right.} \\
& -\left(\delta_{\bar{b}}^{\bar{b}} \delta_{d}^{*} \delta_{\bar{*}}^{\bar{d}} \tilde{D}^{-\bar{c}} \Omega_{c a}{ }_{d}^{\bar{d}} \leftrightarrow_{c}^{\bar{c}}\right)+\left(\delta_{a} \delta_{d}^{*} \delta_{\bar{*}}^{\bar{d}} \tilde{D}_{c} \Omega^{\bar{c} \bar{b}}-{ }_{d}^{\bar{d}} \leftrightarrow{ }_{c}^{\bar{c}}\right) \\
& \left.\left.-\left(\delta_{d}^{*} \delta_{\bar{*}}^{\bar{d}} \tilde{D}_{:} \Omega^{\bar{c} \bar{b}} \Omega_{c a}-{ }_{d}^{\bar{d}} \leftrightarrow_{c}^{\bar{c}}\right)\right\} \mathcal{C}^{-{ }_{*}^{*}}\right](\sigma) \delta\left(\sigma-\sigma^{\prime}\right)
\end{aligned}
$$

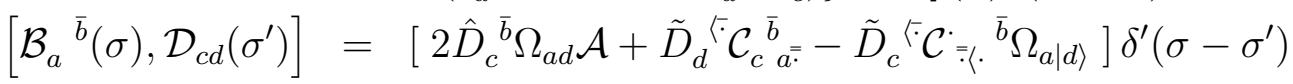

$$
\begin{aligned}
& +\left[4 \nabla \hat{D}_{c}^{\bar{b}} \Omega_{a d} \mathcal{A}-\tilde{D}^{\cdot \bar{*}} \nabla \mathcal{C}_{\overline{\bar{c}_{c}}}{ }^{\bar{b}} \Omega_{a d}\right. \\
& \left.+\tilde{D}_{d}^{\bar{i}}\left\{-\nabla \mathcal{C}_{c a^{-}}^{\bar{b}}+\left(\nabla \mathcal{C}_{c a}^{\cdot} \cdot \mathcal{D}_{c a}\right) \delta_{\cdot}^{\bar{b}}+\left(\frac{1}{2} \nabla \mathcal{C}^{\cdot \bar{b}}{ }_{.=}+\overline{\mathcal{D}}_{\cdot}^{\bar{b}}\right) \Omega_{a c}\right\}\right] \delta\left(\sigma-\sigma^{\prime}\right)
\end{aligned}
$$

It is noted that the covariant derivatives $\nabla_{\sigma}$ are not usual covariant derivatives on constraints, but $\nabla_{\sigma}$ acts on parameters as usual $S p(4)$ covariant derivatives if one multiply parameters to these algebra. Since commutators among $\mathcal{C}$ 's and $\mathcal{D}$ 's always contain two $\hat{D}$ 's, they make closed algebras.

The AdS superstring algebra does not have the form of the AdS superparticle algebra (3.2),$\left.\left(D^{2}\right)_{A}^{B}\right|_{\text {Traceless }}=0$. Because of the stringy anomalous term $[\langle\hat{\mathbf{D}}\rangle,\langle\hat{\mathbf{D}}\rangle] \sim \delta^{\prime}\left(\sigma-\sigma^{\prime}\right)$, the poisson bracket between two $\mathcal{B}$ 's contains $\delta^{\prime}\left(\sigma-\sigma^{\prime}\right) \mathcal{C}$ term which no more has the 
form of (3.2). The number of $\mathcal{C}$ constraints is $120=\left(\begin{array}{c}10 \\ 3\end{array}\right)$ which is the number of degrees of freedom of three forms in 10-dimensions. The total number of this constraints set is $1+16+120+10=147$ which is not $8^{2}-1=63$ of the AdS superparticle case but is same as the flat superstring case [7, 9]. Interesting result is that this first class constraint set $\mathcal{A}=\mathcal{B}=\mathcal{C}=\mathcal{D}=0$ satisfy the same algebra of the one for the flat case [7, 9]. This first class constraint set represents the same as the system with the second class constraints $F=\bar{F}=0$ in (4.3).

\section{$5 \quad$ Conclusions and discussions}

In this paper we have examined the manifest superconformal construction of the AdS superparticle and superstring described by the supermatrix valued coordinates. By introducing supercovariant derivatives whole symmetries of the AdS superparticle are simply described by $D^{2}=0$, adding to $(S p(4) \times G L(1))^{2}$ constraints $(\mathbf{D})=\operatorname{tr} \mathbf{D}=0$. $\kappa$-symmetry and its infinitely reducibility for both the AdS superparticle/string are shown as that half of each $\Theta$ and $\bar{\Theta}$ can be gauged away. So the complex gauge proposed in [6] can not be applied. Equation of motion of the AdS superparticle is obtained as free null AdS geodesics.

The equation of motion for the AdS superstring is obtained as two-dimensional wave equation with induced AdS connection. The left/right chiral separation from this two dimensional equation is not obvious, but we obtained left/right chiral supercovariant derivative algebra from the action. Chiral separation of the supercovariant derivatives and spacetime local symmetry algebra are possible up to constraints. The origin of this weakly chiral separation can be explained as that the AdS superstring is described by supercovariant derivatives and currents which satisfy inhomogeneous $G L(4 \mid 4)$ rather than just $G L(4 \mid 4)$. In other words stringy extension is not incorporated with the superconformal symmetry $G L(4 \mid 4)$. Local symmetry constraint set for the AdS superstring obtained by direct computation from the action is turned out to be the same with the one for the flat case, $\mathcal{A B C D}$ constraints.

Quantization of these systems are possible in the first class constraints approach for the AdS superparticle and the "light-cone-like" gauge for both the AdS superparticle and string as we have shown. This model is easy to handle as the AdS superstring system, and will be useful toward the AdS superstring correction to the classical AdS supergravity theory.

\section{Acknowledgments}

This research is partially supported by the grant-in-aid for Scientific Research, No.12640258 Ministry of Education Japan. 


\section{References}

[1] L.Maldacena, Adv. Theor. Math. Phys. 2 (1998) 231, hep-th/9711200;

S.S.Gubser, I.R.Klebanov and A.M.Polyakov, Phys.Lett. B248 (1998) 105, hepth/9802109;

E.Witten, Adv. Theor. Math. Phys. 2 (1998) 253, hep-th/9802150.

[2] R.R.Metsaev and A.A.Tseytlin, Nucl. Phys. B533 (1998) 109, hep-th/9805028;

Phys. Lett. B436 (1998) 281, hep-th/9806095.

[3] R.Kallosh, J.Rahmfeld and A.Rajaraman, J. High Energy Phys. 9809 (1998) 002, hep-th/9805217;

R.Kallosh and J.Rahmfeld, Phys. Lett. B443 (1998) 143, hep-th/9808038;

J.Rahmfeld and A.Rajaraman, Phys. Rev. D60 (1999) 64014, hep-th/9809164;

I.Pesando, J. High Energy Phys. 9811 (1998) 002, hep-th/9808020;

R.Kallosh and A.A.Tseytlin, J. High Energy Phys. 9810 (1998) 016 hep-th/9808088.

[4] A.Rajaraman and M.Rozali, Phys. Lett. B468 (1999) 58, hep-th/9902046;

S.Forste, D.Ghoshal and S.Theisen, J. High Energy Phys. 9908 (1999) 0013, hepth/9903042;

N.Drukker, D.J.Gross and A.A.Tseytlin, J. High Energy Phys. 0021 (2000) 0004, hep-th/0001204.

[5] R.R.Metsaev, Class. Quant. Grav.18(2001) 1245, hep-th/0012026;

R.R.Metsaev, C.B.Thorn and A.A.Tseytlin, Nucl. Phys. B 596 (2001) 151, hepth/000917.

[6] R.Roiban and W.Siegel, J. High Energy Phys. 0011 (2000) 024, hep-th/0010104.

[7] W.Siegel, Nucl. Phys. B263 (1985) 93.

[8] W.Siegel, Phys. Lett. B203 (1988) 79.

[9] W.Siegel, "Introduction to string field theory" (World Scientific, Singapore, 1988).

[10] W.Siegel, "Free field equation for everything", in string, Cosmology, Composite Structures (March 11-18,1987, College Park . Mryland), eds. S.T.Gates Jr. and R.N.Mohapatra (World Scientific, Singapore, 1987).

[11] M.Hatsuda, Int. J. Mod. Phys. bf A7 (1992) 1187.

[12] E.Inönü and E.P.Wigner, Proc. Natl. Acad. Sci. U.S.A. 39 (1953) 510,

E.Inönü: in "Group Theoretical Concepts and Methods in Elementary Particle Physics" ed. F.Gursey (Gordon and Breach, New York, 1964).

[13] M.Green and J.H.Schwarz, Nucl. Phys. B 243 (1984) 285;

T.Hori and K.Kamimura, Prog. Theor. Phys. 73 (1985) 476.

[14] K.Kamimura and M.Tatewaki (Hatsuda), Phys. Lett. B205 (1988) 257;

A.R.Mikovi'c and W.Siegel, Phys. Rev. B209 (1988) 47.

[15] F.Essler, E.Laenen, W.Siegel and J.P.Yamron, Phys. Lett. B254 (1991) 411;

F.Essler, M.Hatsuda, E.Laenen, W.Siegel and J.P.Yamron, Nucl. Phys. B364 (1991) 67. 